\title{
The second eye of Japanese patients with unilateral exudative age related macular degeneration
}

Masanobu Uyama, Kanji Takahashi, Noriko Ida, Miki Miyashiro, Akira Ando, Ai Takahashi, Eri Yamada, Junya Shirasu, Yoshimi Nagai, Masamitsu Takeuchi

\begin{abstract}
Aim-To clarify the incidence of choroidal neovascularisation (CNV) and predisposing findings for development of CNV in the second eye of Japanese patients with unilateral exudative age related macular degeneration (AMD).
\end{abstract}

Methods-The second eyes of unilaterally affected patients with exudative (neovascular) AMD treated in our clinic during the past 10 years (1988-97) were carefully followed up for more than a year. Evidence of CNV was confirmed by fluorescein and indocyanine green angiography. Macular lesions in patients, in whom CNV developed in the second eye, were retrospectively evaluated from patient records. Results -170 patients met the criteria. The average follow up period was 47 months (range 12-108 months). All patients were Japanese. CNV developed in the second eye in $12(7 \%)$ of 170 patients, 30.3 months on average after the first examination. Cumulative incidence of developing $\mathrm{CNV}$ in the second eye using Kaplan-Meier life table analysis was: $0.6 \%$ by 1 year, $5.6 \%$ by 3 years, and $12.3 \%$ by 5 years, and was relatively low compared with that in white patients. CNV developed most frequently from serous pigment epithelial detachment (PED) in the macula (58\%). Soft drusen were not prevalent and risk of developing CNV was not very high (18\%).

Conclusion-It was confirmed that there were some differences in the incidence and predisposing findings for CNV developing in AMD among Japanese and other Asian patients compared with those in white people. It is important to recognise these differences between the two populations to understand the pathogenesis and epidemiology of AMD.

(Br f Ophthalmol 2000;84:1018-1023)

Exudative (neovascular) age related macular degeneration (AMD) is a leading cause of blindness in elderly people in Western countries. ${ }^{1-4}$ Although, in Japanese as well as in other Asian people, the number of patients with exudative AMD is not as great as in Western people, the number of patients has been rapidly increasing recently and is now becoming one of the major causes of blindness in the elderly. Epidemiology and clinical features of exudative AMD have been well established in the United States and United Kingdom. In
Japan, clinical manifestations of AMD have become clearer during the past 20 years. ${ }^{5}{ }^{6}$ Following the increase in patients with AMD, we have recognised that Japanese patients show some differences from white patients with regard to epidemiological features and predisposing findings for the development of choroidal neovascularisation $(\mathrm{CNV}) .{ }^{56}$

In white people, soft drusen at the macula prevalent among elderly people, are commonly present in AMD, and these people show the highest risk for developing CNV. ${ }^{1-4}{ }^{7-17}$ In Japanese people, however, soft drusen are not as commonly seen among elderly people, or among patients with AMD but, rather, serous retinal pigment epithelial detachments (PED) are the most frequent predisposing lesions for developing CNV. ${ }^{5}{ }^{18}$ These findings were also similar to those in other Asian patients. ${ }^{19}$

Many papers have reported a very high annual incidence of CNV in the second eye in exudative AMD among Western patients. $^{1-4}$ 7-14 2021 We, however, have noted that in Japanese and Asian patients the incidence is not as high. ${ }^{18}{ }^{19}$ It is important to confirm these differences between the two groups, to understand the aetiology and pathogenesis of AMD. Our clinic is one of the leading centres for AMD and macular diseases in Japan, many patients with early and late AMD are referred to us, and we have collected a large series of AMD patients. We attempted to evaluate macular lesions and the development of CNV in the second eyes of Japanese patients with unilateral exudative AMD.

\section{Patients and methods}

The second eyes of unilaterally affected patients with exudative (neovascular) AMD were followed up in our clinic for the last 10 years (from 1988 to 1997), and were carefully examined by one of the two authors who are experienced specialists (MU or KT). Routine ophthalmological examination, direct and indirect binocular ophthalmoscopy, slit lamp biomicroscopy with a contact lens, fluorescein fundus angiography, and indocyanine green (ICG) angiography were performed in all patients. Macular manifestations were retrospectively reviewed from patient records. The patients were regularly followed up at 1-3 month intervals, and whenever a patient presented with any new symptom.

Using procedures described above, it was confirmed that the second eye did not show any evidence of CNV at the first examination. Dur- 


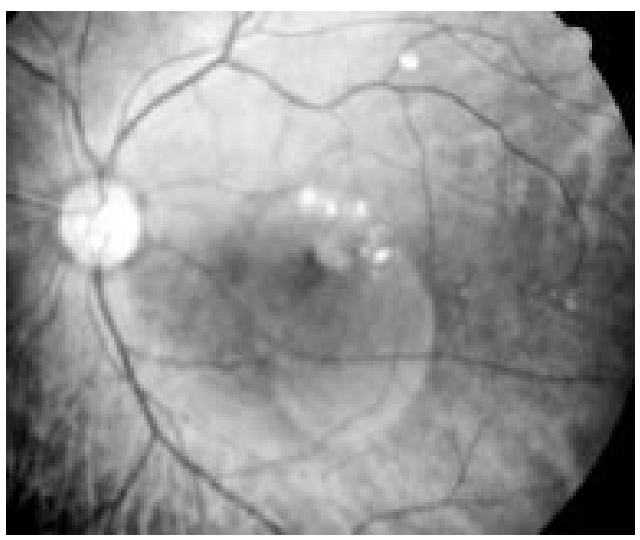

Figure 1 Fundus photograph of serous retinal detachment associated with a small nodular yellowish-white lesion (fibrovascular membrane), tiny haemorrhage, and lipid deposits. A typical macular manifestation in the early stage of exudative age related macular degeneration.

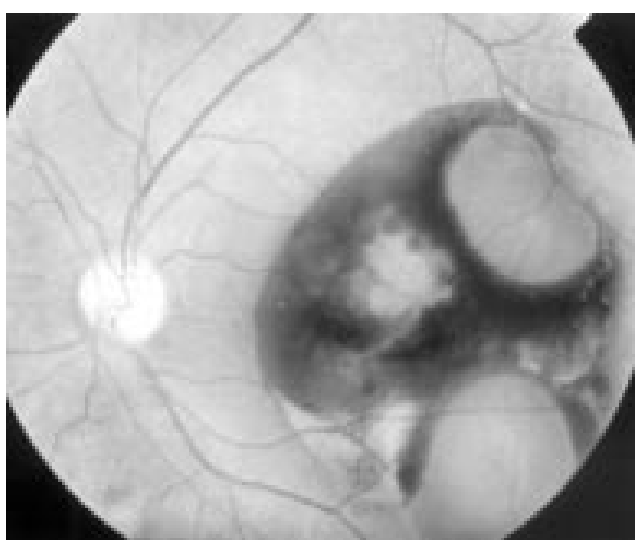

Figure 2 Fundus photograph of subretinal and subpigment epithelial haemorrhage (haemorrhagic pigment epithelial detachment, PED). A typical manifestation of exudative AMD.

ing the follow up period development of $\mathrm{CNV}$ in the second eye was confirmed by fluorescein angiography and/or ICG angiography, when findings in the macula showed any sign of $\mathrm{CNV}$ (haemorrhage, exudation, or elevation).

INCLUSION CRITERIA FOR EXUDATIVE AMD IN THIS SERIES

Patients were more than 50 years of age. The macula showed serous and/or haemorrhagic retinal detachment, with or without serous and/or haemorrhagic retinal pigment epithelial detachments (PEDs), subretinal fibrinous exudations with subretinal fibrovascular membrane, or subretinal fibrous scarring in the macula. Fluorescein and/or ICG angiography clearly demonstrated evidence of CNV, both classic and occult types, in the macula. CNV was confirmed as the following standard with fluorescein angiography:

Classic $C N V$ - In the early phase, lacy hyperfluorescence with a well demarcated margin, and in the late phase progressive leakage from them.

Occult CNV-Ill defined early hyperfluorescence and late leakage. On ICG angiography, early network of hyperfluorescence and late subretinal staining were demonstrated.

Exclusion criteria were patients with other causes of exudative maculopathy such as

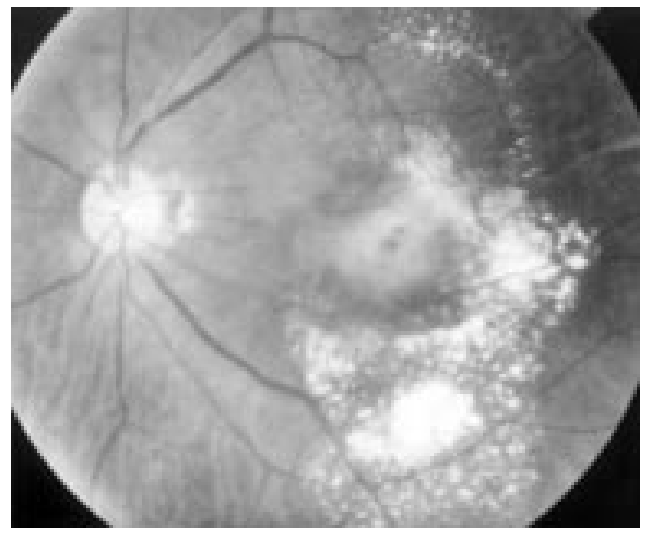

Figure 3 Fundus photograph of subretinal fibrinous exudation and fibrovascular membrane with serous retinal detachment, subretinal haemorrhage, and lipid deposits. This lesion is a so called disciform lesion, and one of the typical manifestations of exudative AMD.

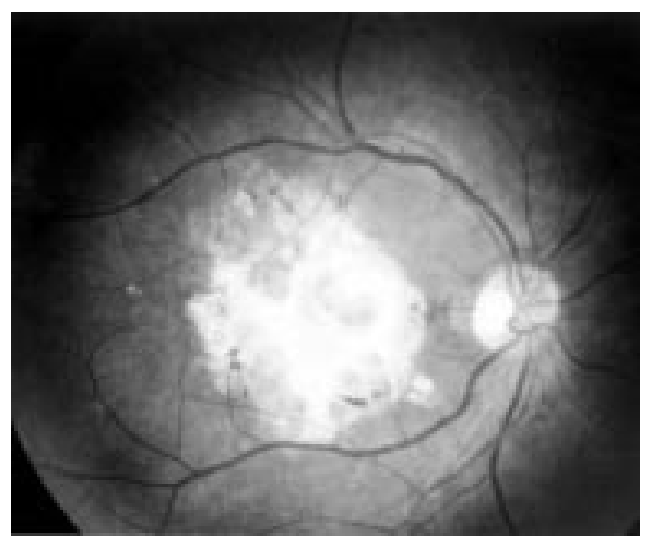

Figure 4 Fundus photograph of subretinal fibrous scar. This lesion is the end stage of exudative AMD.

angioid streaks, high myopia, presumed ocular histoplasmosis syndrome, idiopathic polypoidal choroidal vasculopathy, and central serous chorioretinopathy, follow up period less than 1 year, lack of regular follow up, and incomplete patient records.

Macular manifestations, such as hard drusen, soft drusen, serous and haemorrhagic PED, and retinal pigment epithelium (RPE) abnormality (pigmentary degeneration, RPE atrophy and degeneration) were defined according to the international classification and grading $^{22}$ as follows. Hard drusen are small, round, sharply margined, and yellowish white deposits at the level of RPE. Soft drusen are large, fuzzy bordered, solid, thick in appearance, and yellowish grey deposits at the deep retina. Some are confluent and domeshaped.

Pigment epithelial detachment (PED) - Serous PED is round or oval, yellow or yellow brown, sharply demarcated mound of elevated RPE, without evidence of $\mathrm{CNV}$ on angiography. Haemorrhagic PED contains bleeding from $\mathrm{CNV}$, showing a hyphaema-like horizontal line and a dark elevated mound under the retina. RPE abnormalities - RPE at the macula shows brownish degeneration, or an atrophic appearance, or a small area of mottled pigmentation, hyperpigmentation, or hypopigmentation. 

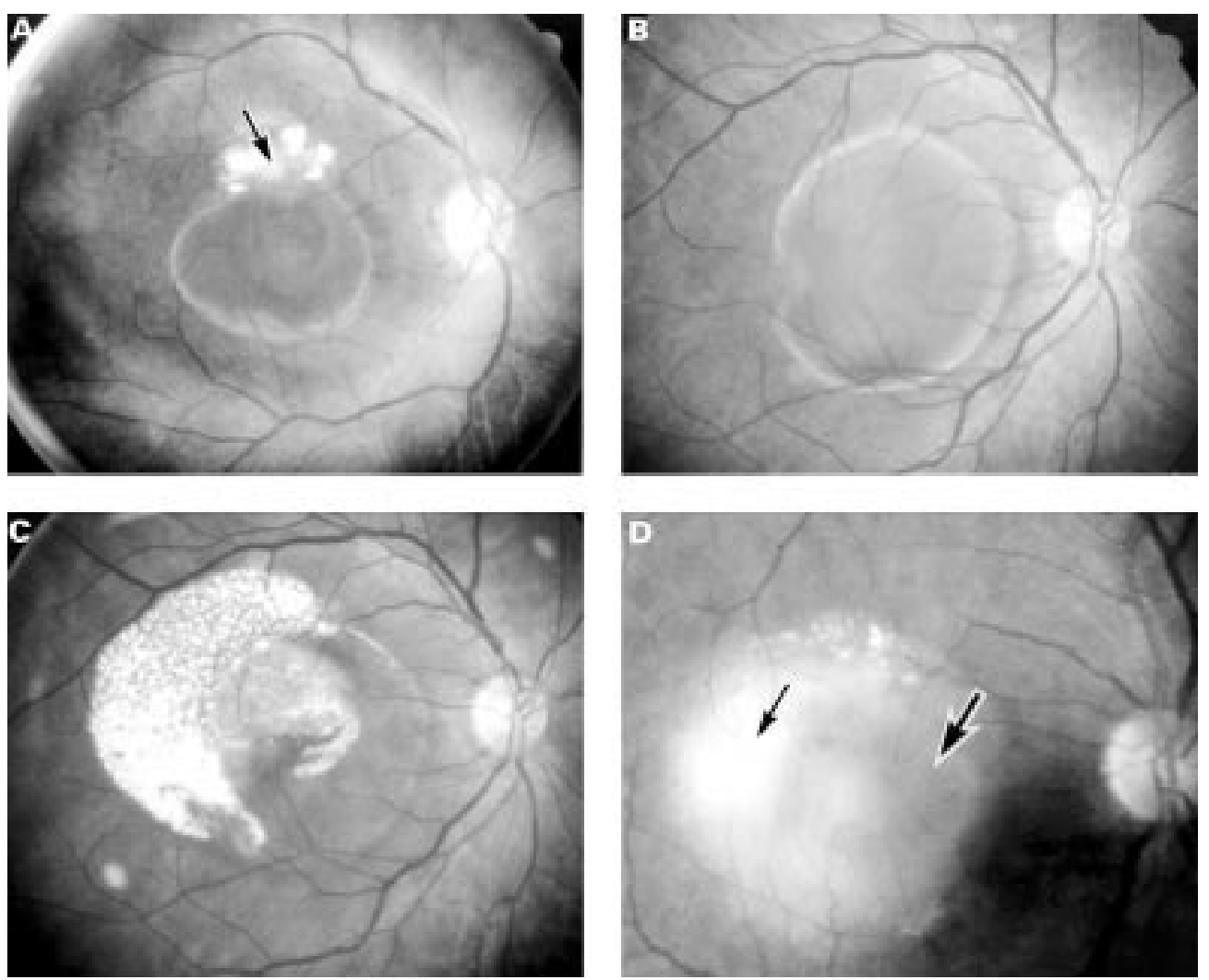

Figure 5 Exudative AMD derived from predisposing a serous PED. (A) Fundus photograph of a large serous PED accompanied by a small fibrovascular membrane (arrow) and lipid deposits. (B) Fundus photograph of haemorrhagic PED. A large serous PED contained bleeding at the bottom of the PED. (C) Fundus photograph of a large serous PED associated with serous retinal detachment and lipid deposits. (D) Fundus photograph of serous PED (large arrow) associated with subretinal exudation, serous retinal detachment, and subretinal fibrovascular membrane (small arrow).

\section{Results}

During the last 10 years, 170 patients with unilateral exudative AMD met the criteria described above. The average follow up period was 42 months, ranging from 12 to 108 months. There were 110 men $(65 \%)$ and 60 women $(35 \%)$. The average age of these patients was 67 years of age, ranging from 44 to 87 , at the first examination. All patients were Japanese.

Macular lesions of the affected eyes of 170 patients at the first examination were as follows. The most frequently seen finding in the involved eyes was serous neurosensory retinal detachment with a small nodular yellowishwhite lesion (fibrovascular membrane) at the macula associated with tiny subretinal haemorrhage (Fig 1). These findings were seen in 81 eyes $(48 \%)$.

Secondly, large dense subretinal and/or subpigment epithelial haemorrhages were seen at the macula in 36 eyes (21\%) (Fig 2). Massive subretinal fibrinous exudation with subretinal fibrovascular membrane, subretinal haemorrhage and serous retinal detachment, which is a

Table 1 Macular lesions of the fellow eye in unilateral exudative $A M D$ at the first examination (170 eyes)

\begin{tabular}{ll}
\hline Findings in the macula & No of eyes \\
\hline RPE abnormality & $52(30 \%)$ \\
Hard drusen & $38(22 \%)$ \\
Soft drusen & $11(6 \%)$ \\
Serous PED & $8(5 \%)$ \\
No abnormality & $83(49 \%)$ \\
\hline
\end{tabular}

typical manifestation of exudative AMD and described as a disciform lesion, were seen in 30 eyes $(18 \%)$ (Fig 3). Subretinal fibrous scarring was seen in two eyes (1\%) (Fig 4). Large serous and/or haemorrhagic PEDs associated with serous retinal detachment were seen in 22 eyes (13\%) (Fig 5A-D).

Macular lesions of the second eye at the first examination (baseline) are shown in Table 1. The most frequently seen finding was RPE abnormalities in 52 eyes $(30 \%)$ (Fig 6). Soft drusens were seen in only 11 eyes (6\%) (Figs 7,

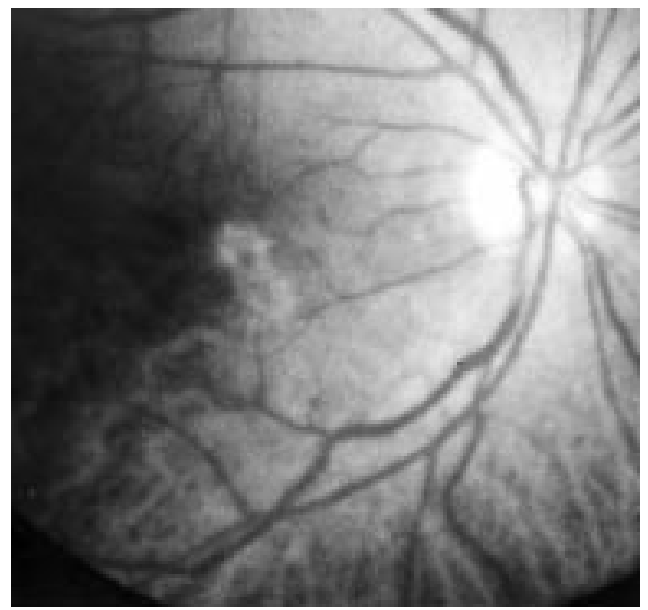

Figure 6 Fundus photograph of degeneration of the retinal pigment epithelium (RPE) at the macula. This is one of the predisposing findings for developing choroidal neovascularisation (CNV). 


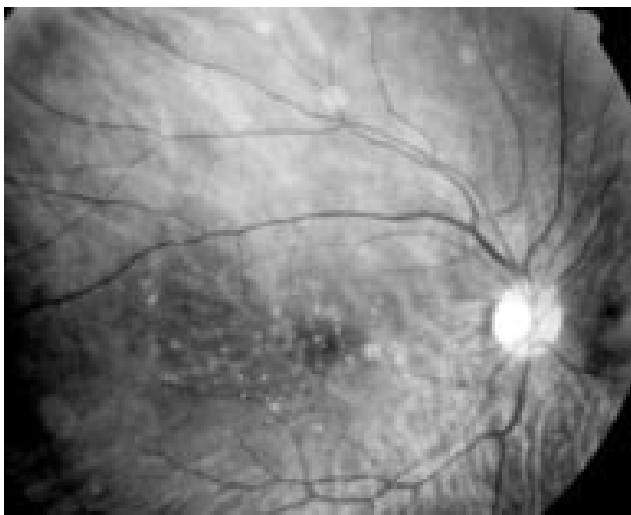

Figure 7 Fundus photograph of hard drusen, seen in elderly patients.

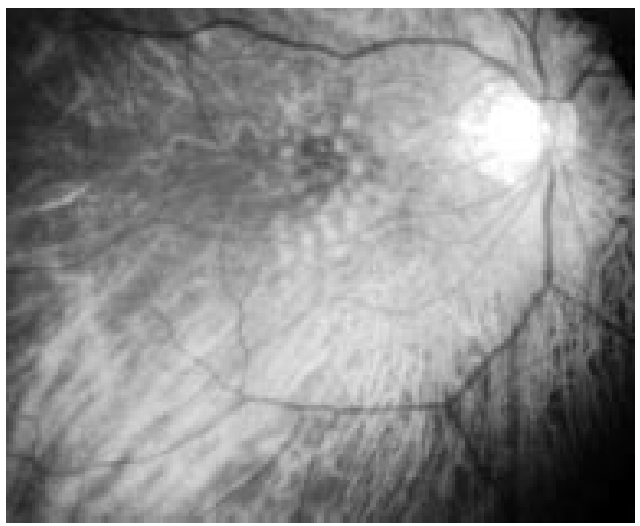

Figure 8 Fundus photograph of soft drusen.

8, 9). Serous PEDs were seen in eight (5\%) (Fig 10). Half of the eyes (49\%) showed no abnormality in the macula.

During the follow up period, CNV, newly developed in the second eye, was seen in 12 $(7 \%)$ of 170 eyes, at an average of 30.3 months (12-59 months), after the first examination (Table 2). The patient age in these 12 cases was 70.8 years on average, ranging from 58 to 82 . There were eight men and four women. The cumulative incidence of the development of CNVs was estimated using the Kaplan-Meier life table analysis. Cumulative incidence of developing $\mathrm{CNV}$ in the second eye was $0.6 \%$ at 1 year, $5.6 \%$ at 3 years, and $12.3 \%$ at 5 years (Fig 11).

Macular lesions from which CNV developed in the second eye of these 12 patients are shown in Table 3 . In these 12 eyes, the most frequently seen predisposing manifestation was serous PED, which was seen in seven eyes (58\%) (Fig 10). The size of PED in which CNV developed varied from 0.3 disc diameter (DD) to $1.5 \mathrm{DD}$, and was $0.6 \mathrm{DD}$ on average. Of these seven eyes with serous PED it was pre-existing at the first examination in one eye. Among the other six eyes, serous PED developed from soft drusen in one eye, from hard drusen in two eyes, from RPE abnormalities in two eyes, and there was no macular abnormality at the first examination in one eye. These serous PEDs in which CNV developed spontaneously and suddenly appeared from various manifestations at the macula. There-

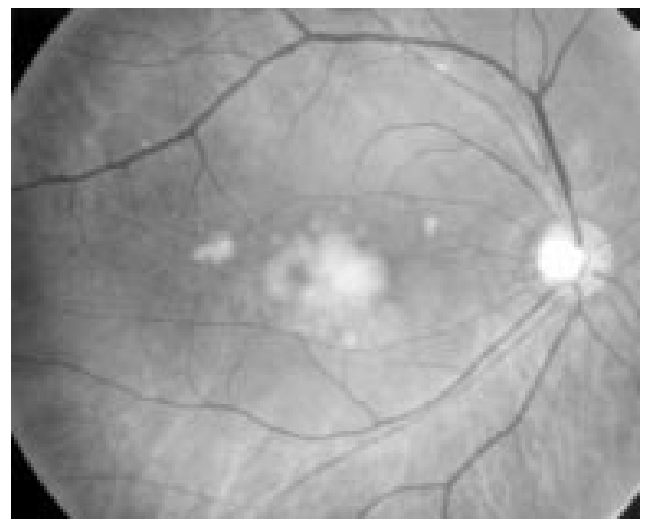

Figure 9 Fundus photograph of large confluent soft drusen. This is a typical predisposing finding of developing CNV. In the elderly, particularly in white people with $A M D$, they are very prevalent, but in fapanese they are not so prevalent.

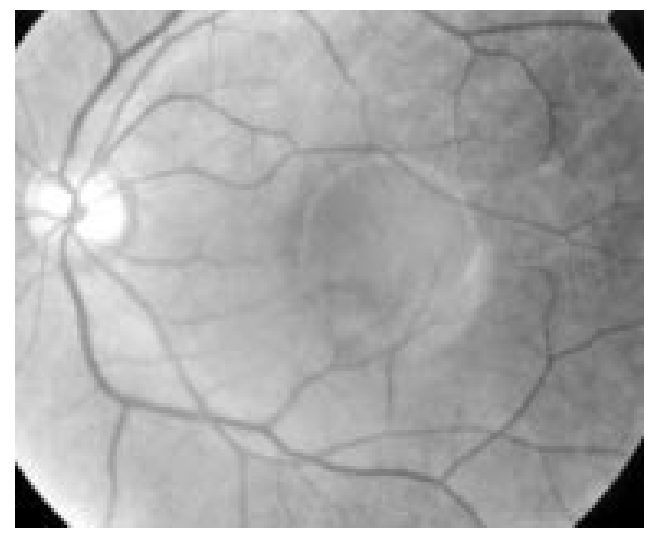

Figure 10 Fundus photograph of a serous PED. This lesion is the commonest predisposing finding for developing $C N V$ in fapanese. CNV develops inside or beside serous PED.

after, CNV usually developed in a short period within or adjacent to the serous PEDs.

CNV developed from soft drusen in two eyes (18\%) (Fig 8). In these two eyes, many large soft drusen were present at the first examination (Fig 9); thereafter CNV developed within these lesions. CNV developed from RPE abnormalities in three eyes (25\%) (Fig 6), (Table 3).

After developing CNV, most eyes showed serous detachment of the neurosensory retina in the macula with a small fibrovascular membrane (Fig 1), and the remaining eyes had subretinal haemorrhage (Fig 2), or serous PED associated with serous retinal detachment and fibrovascular membrane (Fig 5A-D).

\section{Discussion}

The incidence of CNV in the second eye of white patients with unilateral exudative AMD was reported to be high. Many studies reported the incidence of developing CNV in the second eye were $12-15 \%$ per year, ${ }^{10} 5-10 \%$ per year, ${ }^{7}$ $3-15 \%$ per year ${ }^{13}$; the cumulative incidence of developing CNV was estimated as $13 \%$ at 1 year, $22 \%$ at 2 years, and $29 \%$ at 3 years ${ }^{11} ; 3 \%$ at 1 year, $10 \%$ at 2 years, and $20 \%$ at 3 years ${ }^{13}$; $4 \%$ at 1 year, $10 \%$ at 2 years, and $17 \%$ at 3 years. ${ }^{12}$ These were retrospective studies. 
Table 2 Patients developing CNV in the second eye

\begin{tabular}{lllll}
\hline Patient No & Age/sex & $\begin{array}{l}\text { Interval (months) until } \\
\text { developing CNV after first } \\
\text { examination }\end{array}$ & $\begin{array}{l}\text { Macular lesion from } \\
\text { which CNV developed }\end{array}$ & $\begin{array}{l}\text { Size of PED } \\
\text { (DD) }\end{array}$ \\
\hline 1 & $82 \mathrm{M}$ & 12.0 & soft drusen & \\
2 & $72 \mathrm{~F}$ & 13.5 & serous PED & 1.5 \\
3 & $82 \mathrm{~F}$ & 17.7 & soft drusen & \\
4 & $79 \mathrm{M}$ & 20.0 & RPE abnormality & \\
5 & $59 \mathrm{M}$ & 21.6 & RPE abnormality & \\
6 & $76 \mathrm{M}$ & 26.0 & serous PED & 0.8 \\
7 & $62 \mathrm{M}$ & 27.1 & serous PED & 0.3 \\
8 & $66 \mathrm{M}$ & 29.8 & serous PED & 0.5 \\
9 & $81 \mathrm{M}$ & 42.0 & RPE abnormality & \\
10 & $70 \mathrm{M}$ & 44.5 & serous PED & 0.4 \\
11 & $58 \mathrm{~F}$ & 51.0 & serous PED & 0.3 \\
12 & $62 \mathrm{~F}$ & 58.8 & serous PED & 0.5 \\
mean & 70.8 & 30.3 & & 0.6 \\
\hline
\end{tabular}

$\mathrm{CNV}=$ choroidal neovascularisation.

$\mathrm{RPE}=$ retinal pigment epithelium .

PED $=$ pigment epithelium detachment

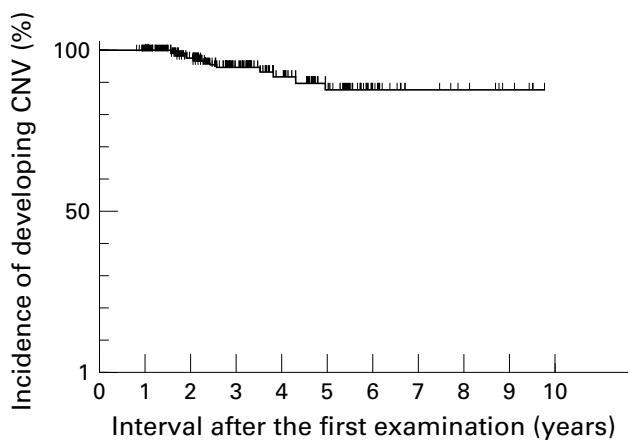

Figure 11 Kaplan-Meier survival curve for development of $C N V$ in the second eye in unilateral exudative $A M D$ (170 eyes).

Table 3 Macular manifestations of the second eyes in which choroidal neovascularisation developed

\begin{tabular}{lll}
$\begin{array}{l}\text { Manifestation in the } \\
\text { macula }\end{array}$ & $\begin{array}{l}\text { No of eyes at the } \\
\text { first examination }\end{array}$ & $\begin{array}{l}\text { No of eyes from which } \\
\text { CNV developed }\end{array}$ \\
\hline Serous PED & 1 & $7(58 \%)$ \\
Soft drusen & 3 & $2(18 \%)$ \\
Hard drusen & 3 & 0 \\
RPE abnormality & 7 & $3(25 \%)$ \\
No abnormality & 1 & 0 \\
\hline
\end{tabular}

$\mathrm{CNV}=$ choroidal neovascularisation

PED = pigment epithelial detachment

$\mathrm{RPE}=$ retinal pigment epithelium.

Prospective studies estimated the incidence was $6 \%$ per year, ${ }^{15}$ and $8.8 \%$ each year. ${ }^{16}$ Moreover, the MPS report ${ }^{23}$ mentioned four independent risk factors for developing $\mathrm{CNV}$ - focal hyperpigmentation, large drusen, multiple drusen, and systemic hypertension. CNV developed in $35 \%$ within 5 years with the cumulative incidence of $10 \%, 28 \%$, and $42 \%$ at 1,3 , and 5 years, respectively. A recent population based study (Beaver Dam Eye Study ${ }^{17}$ ) reported $0.6 \%$ in intact eyes for a 5 year period, a marked increase in patients more than 75 years in age, and a high risk in soft drusen and pigmentary abnormalities at the macula.

Yuzawa $e t a l^{18}$ reported that CNV developed in the second eye in $19(10.4 \%)$ of 183 Japanese patients with unilateral exudative AMD during a 52 months follow up period, and estimated the cumulative incidence of CNV was $3 \%$ at 1 year, $8 \%$ at 3 years, and $12 \%$ at 5 years. Our results showed the incidence of $\mathrm{CNV}$ in the second eye was almost consistent with that reported by Yuzawa et al, and demonstrated a relatively low incidence of CNV developing in Japanese patients compared with white people.

The present study was performed retrospectively using patient records. Patients did not return for regular check ups and incomplete records were excluded. Incidence of developing CNV in the fellow eye of unilaterally involved AMD was estimated on the basis of referral to an ophthalmic clinic. Thus, there was some bias and limitations of evidence, but the low incidence of developing $\mathrm{CNV}$ in the fellow eye of unilateral exudative AMD in Japanese patients was distinct. These results suggested the low prevalence of exudative AMD in Japanese people.

Regarding predisposing findings in the macula for the development of $\mathrm{CNV}$ in $\mathrm{AMD}$ patients, many reports ${ }^{1-4-17}$ described the marked prevalence of soft drusen in elderly white people, indicated that soft drusen showed the highest risk for developing CNV, and that large and confluent soft drusen showed a particularly high risk. In Japanese patients, soft drusen were not as prevalent $(6 \%)$ as in white people, and not a high risk (18\%) for developing CNV compared with white people. These results were consistent with reports ${ }^{18} 19$ on pigmented and Asian people. Formation of soft drusen derives from age related alternations in the RPE and Bruch's membrane. However, the reasons why soft drusen were not as prevalent in these populations as that in white people are not clear.

In white people, reports on risk factors for developing $\mathrm{CNV}$ also indicated that focal hyperpigmentation of the RPE was a high risk. ${ }^{2-4}$ Our result indicated that incidence of CNV from RPE abnormalities was 25\% and at the second rank of predisposing findings in Japanese patients.

Serous PED was the highest risk (58\%) for developing $\mathrm{CNV}$ in Japanese patients, and CNV developed from any size of serous PED. Gass $^{1}$ and Teeters and Bird ${ }^{8}$ described serous PED in the elderly as a predisposing finding for developing CNV. Meredith et $a l^{24}$ reported that in 50 eyes with serous PED, 14 eyes $(28 \%)$ developed CNV at an average of 22 months of follow up, and there was greater risk in eyes with serous PED larger than 1.0 disc diameters and in patients older than 55 years. Braunstein and Gass, ${ }^{25}$ Casswell et $a l,{ }^{26}$ Elman et $a l,{ }^{13}$ and Poliner et $a l^{27}$ confirmed a similar risk in serous PED. Maguire ${ }^{28}$ described the incidence of CNV in patients presenting with only serous PED was estimated to be approximately $40 \%-$ $50 \%$ at 3 years.

Yuzawa et $a l^{18}$ reported that serous PED showed a great risk for developing CNV (30\%) and a much higher risk than that of soft drusen $(4.8 \%)$ in Japanese. Lim et $a l^{19}$ recently reported on symptomatic patients with AMD and found a high percentage of serous PED in Asian patients.

In Japanese and Asian patients with unilateral exudative AMD the incidence of developing CNV in the second eye was relatively low. The predisposing finding indicating the great- 
est risk for developing CNV was serous PED, while soft drusen were not as prevalent and not as high risk compared with that in white people. Serous PED is caused by accumulation of fluid under the RPE, and is derived from damage to fluid movement from the subretinal space through Bruch's membrane. There may be a reduction in adhesive force between the basement membrane and plasma membrane of the RPE and also impairment of electrical conductivity as a result of hydrophobic lipid metabolism in Bruch's membrane. ${ }^{29}$ However, the reason why serous PED is prevalent and soft drusen is not prevalent in elderly Japanese and other Asian patients with AMD is not clear.

In $\mathrm{AMD}$, it was pointed that there were many differences in epidemiology between white people and Asians, as well as in black people. ${ }^{1-6}{ }^{17}{ }^{19}$ In Asian people, AMD is not as prevalent as in white people, AMD in Asian patients was more prevalent in men than in women. In relation to serous PED, central serous chorioretinopathy is prevalent in Asian people compared with in white people. To confirm these differences, further population based prospective studies are essential.

Development of choroidal neovascularisation in exudative AMD may be associated with many factors such as food, smoking, life style, environment, and genetic background. ${ }^{1-4} \mathrm{Al}-$ though the reason for differences in the incidence of new CNV and predisposing findings in AMD between white people and Japanese is unclear, it seems that the difference between these two populations above may be mostly genetic.

In conclusion, the incidence of developing $\mathrm{CNV}$ in the second eye of unilateral exudative AMD was lower in Japanese patients than in white people, showing $12 \%$ at 5 years on a cumulative life table analysis. Regarding predisposing findings for exudative AMD, there were some differences between the two populations. Serous PED was the highest risk factor in Japanese patients among findings predisposing towards the development of CNV. Soft drusen were not as prevalent in Japanese AMD patients and were not a high risk for development of CNV.

This study was supported in part by a grant in aid for scientific research from the Ministry of Education, Science and Culture of Japan (Dr Uyama).

1 Gass JDM. Stereoscopic atlas of macular diseases. 4th ed. St Louis: Mosby, 1997;1: 70-105.

2 Elman MJ, Fine SL. Exudative age-related macular degeneration. In: Ryan SJ, ed. Retina. 2nd ed. St Louis: degeneration. In: Ryan
Mosby, 1994;2:1103-5.

3 Lowenstein A, Bressler NM. Neovascular (exudative) age-related macular degeneration. In: Guyer DR, Yannuzzi LA, Chang S, et al eds. Retina-vitreous-macula. LA, Chang S, et al eds. Retina-vitre
Philadelphia: WB Saunders, 1998;1:94-121.
4 Lawenstein A, Bressler NH, Bressler SB. Epidemiology of MF, Wolfemsberger TJ, eds. The retinal pigment epithelium. MF, Wolfemsberger TJ, eds. The retinal pigment epi
New York: Oxford University Press, 1998:716-23.

5 Uyama M. Choroidal neovascularization, experimental and clinical study. Acta Soc Ophthalmol fpn 1991;95:1145-80.

6 Uyama M, Fukushima I, Takeuchi M, et al. Age-related macular degeneration, clinical features. In: Shimizu K, ed. Current aspects in ophthalmology. Amsterdam: Excepta Medica 1992;2:947-51.

7 Gass JDM. Drusen and disciform macular detachment and degeneration. Arch Ophthalmol 1973;90:206-17.

8 Teeters VW, Bird AC. The development of neovascularization of senile disciform macular degeneration. $A m \quad f$ Ophthalmol 1973;76:1-18.

9 Chandra SR, Gragoudas ES, Friedman E, et al. Natural history of disciform degeneration of the macula. Am $\mathrm{f}$ Ophthalmol 1974;579-82.

10 Gregor Z, Bird AC, Chisholm IH. Senile disciform macular degeneration in the second eye. Br f Ophthalmol 1977;61: $141-7$.

11 Bressler SB, Bressler NM, Fine SL, et al. Natural course of choroidal neovascular membranes within the foveal avascular zone in senile macular degeneration. Am f Ophthalmol 1982;93:157-63.

12 Strahlman ER, Fine SL, Hillis A. The second eye of patients with senile macular degeneration. Arch Ophthalmol 1983; 101:1191-3.

13 Elman MJ, Fine SL, Murphy RP, et al. The natural history of serous retinal pigment epithlium detachment in patients with age-related macular degeneration. Ophthalmology 1986;93:224-30.

14 Bressler SB, Maguire MG, Bressler NM, et al and The Macular Photocoagulation Study Group. Relationship of drusen and abnormalities of the retinal pigment epithelium to the prognosis of neovascular macular degeneration. Arch Ophthalmol 1990;108:1442-7.

15 Macular Photocoagulation Study Group. Five years follow up of fellow eye of patients with age-related macular degeneration and unilateral extra foveal choroidal neovascularization. Arch Ophthalmol 1993;111:1189-95.

16 Sandberg MA, Weiner A, Miller S, et al. Hisk characteristics of fellow eyes of patients with unilateral neovascular age-related macular degeneration. Ophthalmology 1998; 105:441-7

17 Klein R, Klein BEK, Jensen SC, et al. The five-year incidence and progression of age-related maculopathy. The Beaver Dam Eye Study. Ophthalmology 1997;104:7-21.

18 Yuzawa M, Hagita K, Egawa T, et al. Macular lesions predisposing to senile desciform macular degeneration. $7 p n$ f Ophthalmol 1991;35:87-95.

19 Lim JI, Kwok A, Wilson D. Symptomatic age-related macular degeneration in Asian patients. Retina 1998;18:435-8.

20 Lanchoney DM, Maguire MG, Fine SL. A model of the incidence and consequences of choroidal neovascularization secondary to age-related macular degeneration. Arch Ophthalmol 1998;116:1045-52.

21 Schwartz SG, Guyer DR, Yannuzzi LA. Age-related retinal epithelial detachments. In: Marmor MF, Wolfemsberger TJ, eds. The retinal pigment epithelium. New York: Oxford University Press, 1998:706-14.

22 Bird AC, Bressler NM, Bressler SB, et al. An international classification and grading system for age-related maculopa1995;39:367-74.

23 Macular Photocoagulation Study Group. Risk factors for choroidal neovascularization in the second eye of patients with juxtafoveal or subfoveal choroidal neovascularization secondary to age-related macular degeneration. Arch Ophthalmol 1997;115:741-7.

24 Meredith TA, Braley RE, Aaberg TM. Natural history of serous detachments of the retinal pigment epithelium. Am 7 Ophthalmol 1979;88:643-51.

25 Braunstein RA, Gass JDM. Serous detachments of the retinal pigment epithelium in patients with senile macular disease. Am f Ophthalmol 1979;88:652-60.

26 Casswell AG, Kohen K, Bird AC. Retinal pigment epithelial detachments in the elderly:classification and outcome. $\mathrm{Br} \mathcal{F}$ Ophthalmol 1985;69:397-403.

27 Poliner LS, Olk RJ, Burgess D, et al. Natural history of retinal pigment epithelial detachments in age-related macular degeneration. Ophthalmology 1986;93:543-51.

28 Maguire MG. Natural history. In: Berger JW, Fine SC, Maguire MG, eds. Age-related macular degeneration. St Louis: Mosby, 1999:17-30

29 Bird AC, Marshall J. Retinal pigment epithelial detachment in the elderly. Trans Ophthalmol Soc UK 1986;105:674-82. 